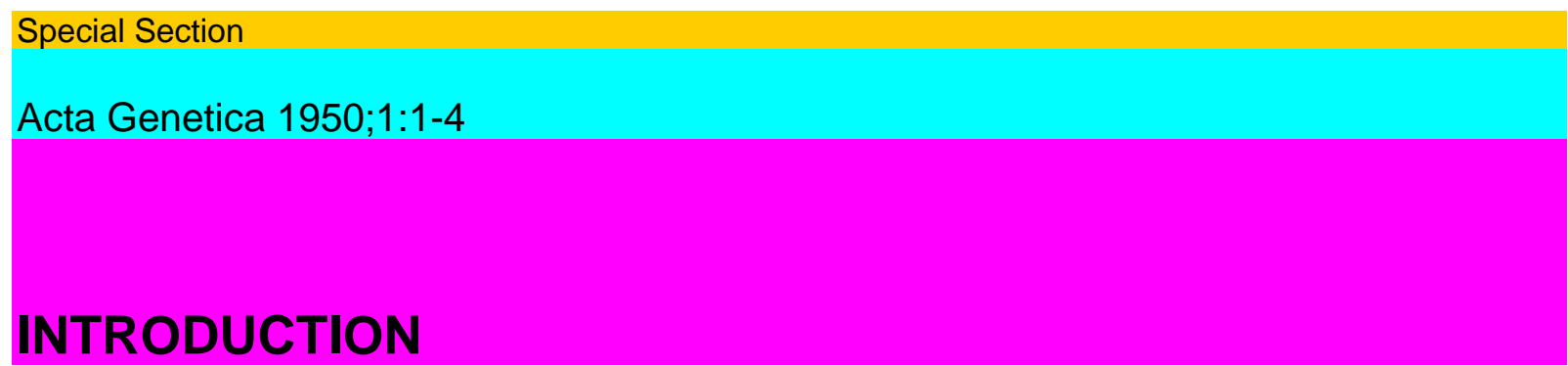

It is natural that, in the world of medicine, it was the environmental factors causing disease which were first dealt with. These, unlike the genetical factors, are comparatively easy to combat. However, before the problems of disease can be fully solved, the genetical factors must also be taken into account, and research into human genetics has gradually come more and more to the fore, until it might well warrant a journal to itself. The genetic investigations on man which have been carried out hitherto have, however, been printed in periodicals of very varying kinds - some in periodicals on genetics, which hardly any medical man reads, and some in medical periodicals, which are not read by geneticists. From this point of view, too, it would be an advantage to have a periodical devoted to Human Genetics.

The solution of problems in this sphere must naturally include the consideration of environmental factors. The diseases are few and, above all, rare that can be considered as purely genetical, í. e. impervious to environmental factors, compared with the relatively large number of diseases where genetical and environmental factors both play a part. Therefore, this periodical will also accept papers bearing on factors of environment, particularly such as take into account the make-up of populations.

That is to say, two fundamental angles can be distinguished in Human Genetics:

What ivill the children of any given marriage be like? We can assume here that we have a more or less extensive knowledge of the tivo parents and of the characters of their relatives.

What will be the future make-up of a population, given more or less detailed data on its present make-up and on the crossings taking place within it ?

The latter problem is naturally the most important from a social vieivpoínt, and it has also begun to receive more attention in recent times. In both cases it is necessary, for scientific work, to use statistical methods. Consequently, investigations of a statistical nature will be particularly welcome in this periodical, especially - as stated above such as deal with the make-up of populations, though it will also publish investigations more interesting to medicine as regards methods. In other mords, the periodical ivill have a special appeal for medical men, but ivill not neglect the interests of those geneticists who are concerned with human genetics. Gunnar Dahlberg.

Acta Genetica, Vol. I, Fasc. 1 (1948) 1

AVANT-PROPOS

II ñ’est que naturel qúOn se soít dans le domaíne de la médecíne occupé en premier lieu des causes de maladies trouvées dans $\Gamma$ en-tourage tnême du malade. Celles-ci sont facíles à combattre par opposition aux facteurs gênétíques. II est toutefoís nécessaíre, avant de pouvoír résoudre complètement les problèmes de la maladie, d"“en tenír compte et $\widehat{\partial}$ est pourquoí les recherches concernant les loís de Thérédíté humaíne ont progressívement gagné du terrain, au point de justífier aujourd'Iiuí un propre périodíque. Les publications de ce domaíne, díspersées jusquHcí, se trouvaíent publíées soít dans des revues géné-tíques à la portée de peu de médecíns, 
soít dans des périodíques médí-caux que les génétícíens lísaíent rarement. II semble avantageux de ce point de vue de créer un journal consacré à Vetude de Vhérédíté.

La solution des problèmes ínhérents doit naturellement tenír compte des facteurs du milieu. II y a peu et de rares maladies à consí-dérer du point de vue génêtíque seulement, comparêes au relatívement grand nombre de maladies basées à la foís sur des facteurs héréditaires et de Tentourage. Cest pourquoí cette revue se propose également de publier des travaux ay ant trait aux facteurs du milieu et, en partículíer, aux príncípes gênétíques des populations.

Deux questions fondamentales se posent en hêrédité humaíne:

Quels seront les enfants d" "un maríage determine? II est à pré-sumer dans ce cas que nous possédons des connaíssances plus ou moíns completes des père et mere, ainsí que des caractères de la parenté.

Quel sera Гêtat futur á"une population, étant donné des dates plus ou moíns precises relatives à sa structure actuelle et les croísements presents ?

Le second problème - étant des plus importants du point de vue social - a attíré ces derniers temps déjà Гattention des ínvestígateurs. Uêtude des deux cas nécessítent toutefoís des méthodes statístíques.

Pour cette raison des explorations statístíques et, aínsí quíl a été relevé plus haut, en partículíer celles sOccupant de la structure hérédítaíre des populations, seront acceptées avec íntérêt, bien que paraîtront également des travaux íntéressant plus spécíalement le mêdecín. Aínsí le journal se consacrera aussí bien aux médecíns qú'aux ínvestígateurs de Thérêdíté humaíne. Gunnar Dahlberg.

\section{ZUM GELEIT}

Es íst nur natürlích, daß man sích auf dem Gebíete der Medízin zuerst $\tau$ nít den

Krankheitsursachen beschäftígt hat, die man in der Umgebung des Kranken fand. Díese sínd leicht zu bekämpfen ím Vergleích mít den Faktoren der Vererbung. Es müssen aber, bevor die Probleme der Krankheiten vollständíg gelöst werden können, auch die genetíschen Faktoren berücksíchtígt werden; darum rückte die For-schung auf dem Gebíete der menschîíchen Erblíchkeítslehre ímmer mehr in den Vordergrund, und nun scheínt es an der Zeít zu seín eíne eígene Zeítschríft auf díesem Gebíete zu gründen. Alle Publíkatíonen waren bísher weít verstreut und in Zeítschríften der verschíedensten Art gedruckt - eíníge in Zeítschríften für Vererbungsforschung, welche Medíziner kaum gelesen haben, andere in medízíníschen Zeítschríften, welche wíederum Vererbungsforschern nícht zu Gesícht kamen. So scheínt es vorteílhaft, eíne Zeítschríft zu besítzen, die sích der mensch-líchen Erblíchkeítsforschung ivídmet.

Die Lösung der Probleme dieser Art muß natürlích die Beachtung der Umgebungs-Faktoren eínschlíeßen. Die Krankheiten, die allein vom genetíschen Standpunkt aus betrachtet werden können, sínd gering an Zahl und noch dazu selten, verglíchen mít der relativ großen Zahl von Krankheiten, beí denen sowohl genetísche wíe Umgebungs- Faktoren eíne Rolle spíelen. Darum wírd díese Zeítschríft auch Arbeiten ver-öffentlichen, die auf Umgebungs-Faktoren basíeren, besonders solche, die den Bevölkerungs-Aufbau in Rechnung zíehen.

Man kann víelleícht zweí fundamentale Angelpunkte in der mensch-líchen Vererbungslehre bezeíchnen:

Wíe werden die Kinder aus eíner bestímmten Ehe seín? Híer können wír voraussetzen, daß wir eíne mehr oder weníger gute Kenntnís der beiden Eltern und der Charaktere íhrer Verwandten besítzen. 
Wíe wírd die künftíge Zusammensetzung eíner Bevölkerung seín, wenn mehr oder weníger detaíllíerte Daten über íhren gegenwärtígen Aufbau und die dabeí vorkommenden Kreuzungen vorhanden sínd?

Das letztere Problem íst natürlích das víel wichtígere vom sozíalen Gesíchtspunkt aus, und es hat auch schon in neuerer Zeít begonnen, mehr Aufmerksamkeít auf sích zu lenken. In beiden Fallen aber íst es notwendíg, daß die Forschung sích statístíscher Methoden bedient. Darum werden Untersuchungen statístíscher Natur willkommen seín und besonders gem - ivíe oben ausgeführt - solche Arbeiten aufgenommen

$1 *$

werden, die sích mít dem Aufbau von Bevölkerungen beschäftígen; doch werden selbstverständlích auch Forschungen publizíert werden, die mehr die medizinischen Interessen berücksichtígen. Mít anderen Worten, die Zeítschríft wendet sích besonders an Medíziner, wírd aber immer auch den Interessen all der Forscher dienen, die sích mít der menschlíchen Vererbung beschäftígen. Gunnar Dahlberg. 